\title{
Safe Physical Human-Robot Interaction: Measurements, Analysis \& New Insights
}

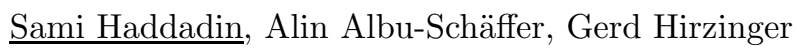

Institute of Robotics and Mechatronics

DLR e.V. - German Aerospace Center

P.O. Box 1116, D-82230 Wessling, Germany

Summary. Physical human-robot interaction and cooperation has become a topic of increasing importance and of major focus in robotics research. Industrial and domestic applications unifying the workspace of humans and robots are foreseeable in the close future and will require safe and dependable robot design and control. In this paper we will give an overview of our systematic evaluation of safety in humanrobot interaction, covering various aspects of major significance.

Based on initial impact tests we carried out with the DLR-LWRIII, several industrial robots of increasing weight were evaluated and the influence of robot mass and velocity was investigated. Such non-constrained impacts are only partially capturing the nature of human-robot safety. A possibly constrained environment and its effect on resulting injuries have to be discussed and evaluated.

Apart from these impact tests and simulations we will analyze the major problem of quasi-static clamping, which poses under certain circumstances a serious threat to the human even for low-inertia robots. After treating blunt impacts and contacts with and without the human being clamped, various soft-tissue injuries likely to occur in robotics and related physical injury tolerances are introduced. Finally, possible injuries relevant in robotics are summarized and systematically classified.

\section{Motivation \& Introduction}

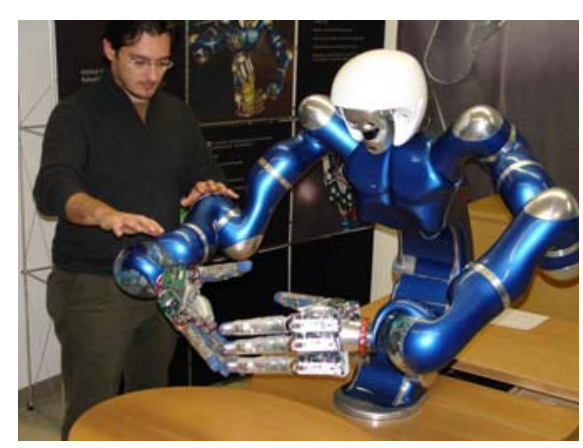

Fig. 1. Physical cooperation between humans and robots.

Bringing robots and humans spatially together as exemplified in Fig. 1 leads to the fundamental concern of how to ensure safety to the human. As 
Asimov already noted very early, safety has priority if robots are close to humans [1]. Intuitively it seems clear that a robot moving at maximal speed (e.g., due to malfunction) can cause high injury, especially if the impact is unexpected. In this sense we will present new results, leading to quite surprising conclusions.

During unexpected collisions, various injury sources are present: fast blunt impacts, dynamic and quasi-static clamping, or cuts by sharp tools. Fundamental work on human-robot impacts under certain worst-case conditions and resulting injuries was carried out in $[2,3,4]$, evaluating free rigid impacts at a robot speed up to $2 \mathrm{~m} / \mathrm{s}$.

From the standardization bodies' side the ISO-10218 was introduced, defining new collaborative operation requirements for industrial robots [5]. It states that one of the following conditions always has to be fulfilled for allowing human-robot interaction: The TCP/flange velocity needs to be $\leq 0.25 \mathrm{~m} / \mathrm{s}$, the maximum dynamic power $\leq 80 \mathrm{~W}$, or the maximum static force $\leq 150 \mathrm{~N}$. In our opinion these requirements tend to be quite restrictive, too undifferentiated and therefore they strongly limit the performance of the robot, as will be supported by our results.

Further important aspects concerning safety in human-robot interaction were evaluated in $[6,7,8]$. However, attempts to investigate real-world threats via impact tests at standardized crash-test facilities and use the outcome to analyze safety issues during physical human-robot interaction were to our knowledge, only carried out in [2] up to now. In order to quantify the potential danger emanating from the DLR lightweight-robot III (LWRIII), impact tests at the Crash-Test Center of the German Automobile Club (ADAC) were conducted and evaluated. The outcome of the dummy crash-tests indicated a very low injury risk with respect to evaluated injury criteria for rigid impacts with the LWRIII. Furthermore, they show that a robot, even with arbitrary mass moving not much faster than $2 \mathrm{~m} / \mathrm{s}$ is not able to become dangerous to a non-clamped human head with respect to typical severity indices ${ }^{1}$. In this paper we will confirm these strong statements by crash-tests with several industrial robots. The experiments will lead us to other injuries which seem more relevant to us in the mentioned case of free impacts. After evaluating free impacts between humans and robots we will analyze dynamic clamping, which is a major source of potential injury especially for massive robots. Apart from such dynamic clamping impacts we identified certain situations in which lowinertia robots as the LWRIII can become seriously dangerous as well. They are related to clamping close to singularities where the robot is able to exert very large external forces.

Soft-tissue injury caused by sharp violence will be discussed and a summary on such injuries and the physical quantities causing them will be given. In the end an overview of possible injuries and related severity measures will be outlined with the goal of assembling a full image of injury mechanisms in robotics which is missing completely in the literature up to now.

The paper is organized as follows. Sec. 3 outlines impact tests with industrial robots and a clamping analysis for blunt dynamic impacts. In Sec. 4 clamping in near-singular configurations is treated and analyzed more in detail, leading to the description of soft-tissue injuries caused by sharp violence in Sec. 5. Fi-

\footnotetext{
${ }^{1}$ Severity indices are injury measures used in the automobile industry. Head injury
} assessing criteria mostly focus on the evaluation of head acceleration. 
nally, a categorization of injuries in robotics is given in Sec. 6 and a conclusion in Sec. 7 summarizes our main achievements.

\section{Initial Impact Tests}

During our initial experiments at the ADAC all standard measurements for automotive crash-tests which can be acquired with a HybridIII-dummy (HIII) for the head, neck and chest were performed. These measurements are presented in detail in [2], where also the exact definition of various indices is given. In this paper we will only present the main conclusions and the lessons learned from these experiments.

Very surprising to us and other robotic specialists (but not for the ADAC staff) was that all evaluated severity indices were located in the lowest quarter of the green area (very low injury) in the EuroNCAP color code (see also HIC plot for LWRIII in Fig. 2). Apart from these results, one is able to draw some further conclusions related to the nature of robot impacts with rigid human body parts such as the head, which to some extend where unexpected, too. They give some new answers to safety questions posed in the robotics literature. Summarized, three main conclusions concerning severity reduction of impact characteristics can be drawn ${ }^{2}$ :

- No physical collision detection mechanism is fast enough to reduce the impact dynamics of fast and rigid impacts.

- For such impacts further joint stiffness reduction does not lower impact forces or severity indices since motor and link inertia are already decoupled.

- Soft covering is an adequate countermeasure to reduce the impact effectively.

Concerning the influence of robot mass and velocity two main statements can be extracted:

- $\mathrm{HIC}^{3}$ saturates with increasing robot mass for each impact velocity.

- Impact velocity is the major factor defining the injury severity.

Especially the first statement, which we gained out of simulation was very surprising to us since it contradicts the intuition of a massive robot being a priori life threatening.

\section{The Effect of Robot Mass and Velocity}

In this section the experimental confirmation of the statements given in Sec. 2 regarding saturation of HIC with robot mass and the possibility of facial and cranial fractures will be evaluated. Furthermore, clamping simulations will be carried out based on measurements with several industrial robots.

\footnotetext{
${ }^{2}$ For explanatory details please refer to [2].

3 The Head Injury Criterion (HIC) is a so-called severity index and is the most prominent indicator of head injury in automobile crash-testing. It was already introduced to the robotics community.
} 


\subsection{Evaluated Robots}

In order to cover a wide range of robots and be able to verify the saturation effect explained in Sec. 2, we compared three industrial robots ${ }^{4}$ with the LWRIII. The tests with the industrial robots were carried out with a a simplified setup, mimicking a HIII head ${ }^{5}$.

A feature of the KR3-SI, which has to be mentioned, is the safeguarding of the tool by means of an intermediate flange with breakaway function, triggering the emergency stop in case the contact force at the TCP exceeds a certain threshold ${ }^{6}$. In combination with the mounted impactor its weight is $1.4 \mathrm{~kg}^{7}$.

\subsection{Head Injury Criterion \& Impact Forces}

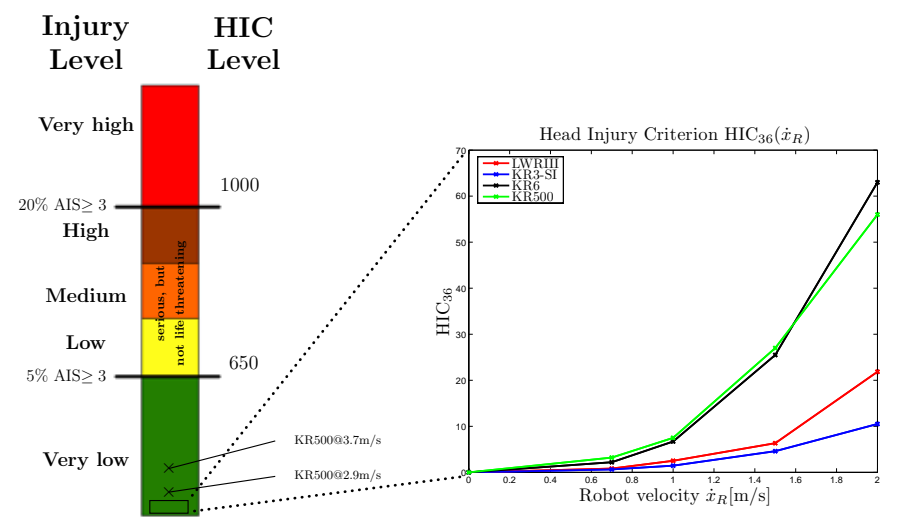

Fig. 2. Resulting $\mathrm{HIC}_{36}$ values at varying impact velocities for all robots, rated according to the EuroNCAP Assessment Protocol And Biomechanical Limits.

In Fig. 2 the resulting $\mathrm{HIC}$ values for the different robots are visualized and classified according to the EuroNCAP. The values for the KR3-SI are even lower than for the LWRIII because the intermediate flange decouples the impactor at the moment of impact from the entire robot. Therefore, only the flange-impactor complex is involved in the impact. Clearly, the saturation effect explained in Sec. 1 is observed, as the numerical values for the KR6 and KR500 do not significantly differ. The simulation results presented in [2] should be considered as conservative, since the actual saturation value is

\footnotetext{
${ }^{4}$ We used the KUKA KR3-SI (54kg), the KUKA KR6 $(235 \mathrm{~kg})$ and the KUKA KR500 (2350kg). The type designations indicate the nominal payload in $[\mathrm{kg}]$ of the robot and the total weight is given in brackets. Reflected inertias in the direction of impact were $\{12,67,1870\} \mathrm{kg}$.

${ }^{5}$ This was due to the high costs of crash-tests at certified facilities. The validity of the setup is shown in [9].

${ }^{6}$ Category 0,1 stop according to DIN EN 60204. Category 0 stop means that the drives are immediately switched off and the brakes engage at the same time. A Category 1 stop lets the robot halt with a hard stop trajectory without using the brakes.

7 A video illustrating many of the aspects in this section is shown in [10] or can be downloaded from www.robotic.dlr.de/safe-robot.
} 

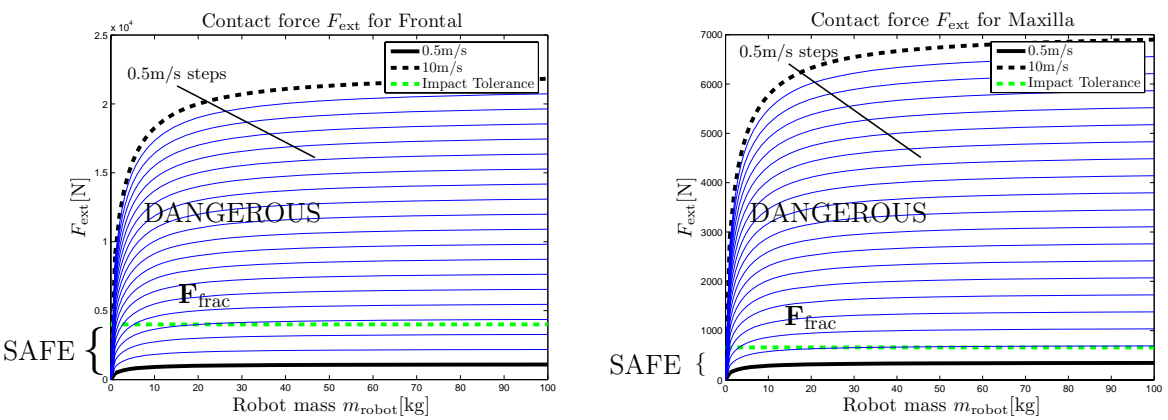

Fig. 3. Contact forces for simulated impacts between a robot and the frontal area (left) and the maxilla (right), showing the dependency on the robot mass and velocity. The impact velocity steps are $0.5 \mathrm{~m} / \mathrm{s}$. One has to take into consideration that the applied human model is not valid anymore after the fracture occurs. This is discussed in [11].

even noticeably lower than predicted by simulation. This means that even an impact of such a huge robot as the KR500 cannot pose a significant threat to the human head by means of typical severity indices from automobile crashtesting. The injury level for these impact tests are as well located in the green area. Even at $2.9 \mathrm{~m} / \mathrm{s}$ and $3.7 \mathrm{~m} / \mathrm{s}$ impact velocity the probability of AIS $\geq 3$ injuries for the KR500 is only $1.2 \%$, respectively $3.6 \%$ as indicated in Fig. 2. HIC and similar criteria are apparently not appropriate measures of possible injuries in robotics, necessitating the investigation of other injury mechanisms like fractions of facial \& cranial bones, possibly occurring during human-robot collisions. This is indicated by recorded contact forces of the impact tests which were in the order of the fracture tolerance of these bones.

In Fig. 3 the dependency of the impact force with respect to the robot mass and velocity (the robot is assumed to move with constant velocity) for the frontal bone and the maxilla are visualized. For all bones ${ }^{8}$ except the frontal one it seems that starting from the saturation mass value ${ }^{9}$, a velocity between $0.5-1.0 \mathrm{~m} / \mathrm{s}$ is enough to cause fractions. The frontal bone is the most resistant one, generally withstanding impacts approximately up to $2 \mathrm{~m} / \mathrm{s}$. Furthermore, it becomes clear that especially for robots with less than $5 \mathrm{~kg}$ reflected inertia at the moment of impact the velocity can be significantly higher without exceeding the limit contact force. For weaker bones like the maxilla impact speeds of $2 \mathrm{~m} / \mathrm{s}$ already pose a major fracture source even for these robots.

\subsection{Facial Impact Forces and Chest Criteria with Clamping}

After analyzing free impacts in detail, the influence of clamping shall now be outlined. In Tab. 1 (left) the clamping forces of the maxilla for impacts with all robots at $2 \mathrm{~m} / \mathrm{s}$ in their particular impact configuration are listed ${ }^{10}$.

\footnotetext{
${ }^{8}$ Simulations for other facial and cranial bones denoted in [9] were carried out as well. They show similar behavior.

9 The robot mass from which on a further increase does not result in significantly higher forces.

${ }^{10}$ For this simulation the KR3-SI is assumed to have no intermediate flange with breakaway function, i.e. we assume a KR3.
} 


\begin{tabular}{lrc}
\hline ROBOT & \multicolumn{2}{c}{ Contact Force Maxilla Fracture? } \\
\hline LWRIII & $0.6 \mathrm{kN} @ 1 \mathrm{~m} / \mathrm{s}$ & No \\
LWRIII & $1.2 \mathrm{kN} @ 2 \mathrm{~m} / \mathrm{s}$ & Yes \\
KR3 & $2.2 \mathrm{kN} @ 2 \mathrm{~m} / \mathrm{s}$ & Yes \\
KR6 (Cat.0\&1) & $5.1 \mathrm{kN} @ 2 \mathrm{~m} / \mathrm{s}$ & Yes \\
KR500 (Cat.0\&1) & $23.6 \mathrm{kN} @ 2 \mathrm{~m} / \mathrm{s}$ & Yes \\
\hline
\end{tabular}

\begin{tabular}{lrrr}
\hline ROBOT & $\mathrm{CC}[\mathrm{mm}]$ & $\mathrm{VC}[\mathrm{m} / \mathrm{s}]$ & $F_{\text {ext }}^{x}[\mathrm{~N}]$ \\
\hline LWRIII & $14.4(0.0)$ & 0.035 & $741.6(1.3)$ \\
KR3 (Cat.0) & $31.2(0.0)$ & 0.1 & $851.9(1.4)$ \\
KR6 (Cat.0) & $65.5(2.0)$ & 0.25 & $2836.1(2.7)$ \\
KR6 (Cat.1) & $66.6(2.1)$ & 0.25 & $2904.6(2.7)$ \\
KR500 (Cat.0) $228.0(6.0)$ & 0.84 & $14282.0(6.0)$ \\
KR500 (Cat.1) & $245.0(6.0)$ & 0.89 & $15491.0(6.0)$ \\
\hline
\end{tabular}

Table 1. Impact forces with clamping at $2 \mathrm{~m} / \mathrm{s}$ obtained for the maxilla (left). Simulated values for chest severity indices and corresponding AIS values at $2 \mathrm{~m} / \mathrm{s}$ obtained for the human chest (right). Cat.0 denotes stopping with brakes and Cat.1 fastest possible stop without brakes.

Each robot reacts to the collision by braking with maximum torque and continuing so until contact with the head is lost. The simulations show the vast influence of the robot mass and already the KR3 produces almost twice the contact force the LWRIII generates ${ }^{11}$. However, all robots, even the low inertia LWRIII potentially break the maxilla at $2 \mathrm{~m} / \mathrm{s}$. Additionally, one has to take into consideration that the applied model is not valid anymore after the fracture occurs because the resistance on the robot is dramatically lowered, possibly causing even more (under certain circumstances even arbitrary) severe secondary injury. However, for the LWRIII a safe velocity of at least $1 \mathrm{~m} / \mathrm{s}$ is possible.

In Tab. 1 (right) the resulting Compression Criterion (CC), the Viscous Criterion (VC) and the clamping forces are listed for impacts at $2 \mathrm{~m} / \mathrm{s}$ with a clamped chest. CC is the deflection of the chest and $\mathrm{VC}$ the relative deflection times intrusion velocity (see [2] for definition). The corresponding EuroNCAP injury level [2] is indicated for the CC and VC by color. For the CC the AIS level is additionally obtained by another available mapping ${ }^{12}$ and is denoted in brackets. The injury level of the CC clearly shows how increasing robot mass leads to a higher probability of injury level. Similar conclusions can be drawn from the contact force and its correlating injury level, showing that both criteria are sensitive predictors of injury for the chest in case of clamping. The Viscous Criterion, in contrast, is due to the low velocities subcritical except for the KR500. This is because even though the intrusion velocity is low, the deflection dominates the VC value in this case. Similar to the head we can see that the chest is exposed to an enormous threat with growing robot mass if the human is clamped. More detailed simulations showing specifically the correlation between impact speed and injury criterion for each robot are as well outlined in [12]. After this investigation of dynamic blunt impacts with and without clamping the problem of quasi-static loading will be discussed as a case-study on the LWRIII, but the resulting methodology of investigation can be applied to any robot.

11 The relation between motor torque and inertia scales disadvantageous when increasing dimensions.

${ }^{12}$ For details please refer to $[2,9]$. 


\section{Singularity Forces during Clamping}

At impact configurations with large levers, robots of similar inertias (and maximum joint torques) to the LWRIII do not pose a potential threat by means of HIC [2], but the almost outstretched arm can be a significant injury threat which now shall be evaluated more in detail, see Fig. 4 (left).

The maximum nominal torques for a given robot are represented by a hyperrectangle. The corners of this hyper-rectangle are then transformed via the pseudo-inverse of the transposed Jacobian to the corners of a hyper-polygon of Cartesian forces. In order to get the maximal applicable force in the relevant worst-case direction, the corresponding hyper-rectangle corner has to be evaluated. Here, we use the collision detection (CD) described in detail in $[13,14]$. Its detection threshold $\tau_{\text {det }}$ for the external joint torque of the robot is defined percentaged to the maximum nominal joint torque $\boldsymbol{\tau}_{\max }$ (e.g., $5 \%$ ) which lets us easily obtain the detection threshold of the contact force.

$$
\boldsymbol{\tau}_{\text {det }}=0.05 \tau_{\max } \rightarrow \mathbf{F}_{\text {det }}=0.05 \mathbf{F}_{\max }=\mathbf{J}^{T \#} \boldsymbol{\tau}_{\text {det }}
$$

$\mathbf{J}^{T \#}$ is the pseudo-inverse of the transposed manipulator Jacobian ${ }^{13}$. To theoretically analyze the configuration boundaries which can cause fractions of facial and cranial bones the reconfiguration from "elbow up" to "elbow down" is the most dangerous case. The robot can be commanded in such a way that it passes the outstretched position if the clamped head is contacted close to the singularity. In Fig. 4 the maximal force which can be exerted on a human maxilla by a rigid, slowly moving robot (no dynamic forces) are analyzed. The stiffness of the maxilla is according to $[15,16,17]$ in the order of $10^{5} \mathrm{~N} / \mathrm{m}$. Thus, the force will linearly increase with position after contact, as represented in Fig. 4 for several collision points along the lines $l_{i}$. The linear forces are displayed only up to the limit at which the bone will break (2), denoted by $\mathbf{F}_{\text {frac }}=660 \mathrm{~N}$. The curve $\mathbf{F}_{\max }$ represents the maximal force that can be exerted by the robot, which goes to infinity when approaching the singularity. If this curve is above $\mathbf{F}_{\text {frac }}$ and if $\mathbf{F}_{\text {frac }}$ is exceeded before reaching the singularity for a given collision point (this depends on the slope of $l_{i}$ ), the bone will break. For the considered case, this would happen starting with (1), i.e. more than $27 \mathrm{~cm}$ before reaching the singularity. Starting with (3), there does not exist even a hypothetical equilibrium point, meaning the considered stiffness cannot stop the robot from reaching the singularity. Using the collision detection with a threshold of $0.05 \tau_{\max }$, the maximal forces are lowered, as displayed by the curve $\boldsymbol{F}_{\text {det }}$. In this case, the critical region is substantially reduced to about $2 \mathrm{~cm}$ before the singularity (4). Restricting the workspace of the arm such that this configuration is not reached, poses no significant limitation to usual applications. The limit safe configuration ${ }^{14}$ is denoted by (5). This analysis can be carried out with all facial and cranial bones listed in [9] and yields similar observations for each of them.

\section{Soft-Tissue Injuries caused by sharp Violence}

Apart from blunt injury mechanisms several soft-tissue injuries caused by sharp violence, which were to our knowledge not treated from a biomechanical point of view in the robotics literature up to now, are very likely to

\footnotetext{
${ }^{13}$ Note, that since the torque $\boldsymbol{\tau}_{\text {det }}$ is produced only by a TCP force, any generalized pseudoinverse will lead to the same value of $\mathbf{F}_{\text {det }}$.

14 With an ideal collision detection and an infinitely fast stopping robot.
} 

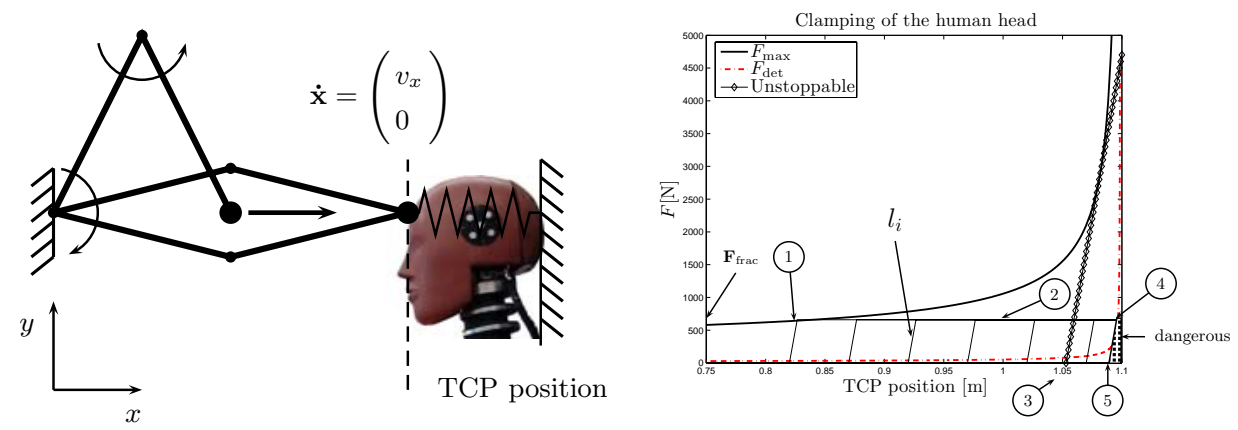

Fig. 4. Clamping the human with a rigid manipulator in near-singular (almost outstretched) configuration, meaning from "elbow up" to "elbow down" or vice versa (left). Theoretical analysis of the reconfiguration of the manipulator (right).

occur during worst-case scenarios in pHRI. When searching for relevant injury mechanisms during physical contact with a robot, such an analysis is the next logical step. Since this topic is still a major part of future research we will give a brief overview on what injury types are worth to focus on next and what tolerance values exist for these injuries.

\subsection{Soft-tissue Injury}

A major potential injury source in pHRI are the various tools a robot can be equipped with and sharp elements along the robotics structure. Their evaluation is still a field with many open issues and definitely worth and fruitful to work on. As a first step one is able to identify the most important injuries and their mechanisms, since investigations were already made in the field of forensic medicine and biomechanics. Therefore, basic statements concerning relevant quantities for different soft-tissue injury mechanisms are possible. According to [18], common and important soft-tissue injuries are

- Abrasion

- Laceration (cuts, gashes, contused wounds)

- Contusion (bruises, crushes)

- Stab wounds

Despite the wide variation among biomechanical analysis and tests, some tolerance values were published in the literature ${ }^{15}$, giving valuable information on which physical quantities potentially lead to and govern different injury mechanism. Abrasions are mainly caused by tangential motions of corners and sharp edges along the human $\operatorname{skin}^{16}$ and an affecting energy of $E \approx 100 \mathrm{~J}$ is already enough to cause such injury. This criterion can directly be transferred to robotics and would cover abrasions caused by structural edges and tools. Contusions, expressed by bruises and crushes, are a matter of impact energy density. Typical regions are the scalp and kneecaps since they have an osseous basis. Tissue injury occurs at an energy density of $e_{A}>2.52 \mathrm{~J} / \mathrm{cm}^{2}$ and haematoma and suffusion already below this value. Tailored to the needs of robotics one will have to transfer these tolerance values to establish safe dynamics, i.e. ensure that these values cannot be exceeded if a collision occurred.

\footnotetext{
${ }_{15}$ A good summary of soft-tissue injury and its tolerance values is given in [18].

${ }^{16}$ The edge is $\approx \perp v_{\text {Robot }}$, otherwise it would be a cut.
} 
Stab wounds were investigated with different knifes and it was concluded that strain is not an appropriate measure to define a tolerance value for knifes and similar tools because the contact area is too small. Instead the evaluation of the penetration force is proposed. Tolerance forces depend on the layers of clothing and range from mean values of $76.5 \mathrm{~N}$ for uncovered skin to $173.2 \mathrm{~N}$ for three layers of typical clothing. The last injury introduced here are lacerations (cuts), which need to be part of this evaluation as well, taking into account the cutting edge of the tool and therefore completing the analysis of tools.

Of course there do exist other injury mechanisms but we believe the presented ones already cover a large fraction of the most probable and important ones which absolutely necessary have to be investigated.

\section{Possible Injuries: Synopsis}

Up to now only isolated issues and mechanisms of robot safety were discussed and introduced in the robotics literature. In order to have an overview of the potential injury threats depending on the current state of the robot and the human, a classification of these mechanisms, governing factors of the particular process and possible injuries are proposed in Fig. 5. Physical contact can be divided into two fundamental subclasses: quasi-static and dynamic loading ${ }^{17}$. Fundamental differences in injury severity and mechanisms are as well observed if a human is (partially) clamped or not, leading to the second subdivision. For the quasi-static case we differentiate between near-singular and non-singular clamping as was outlined earlier. The last differentiation separates injuries caused by blunt contact from the ones induced by tools or sharp surface elements.

Each class of injury is characterized by possible injuries (PI), worst-case factors (WCF) and their worst-case range (WCR). WCF are the main contributors to the worst-case, such as maximum joint torque, the distance to singularity or the robot speed. The worst-case range indicates the maximum possible injury depending on the worst-case factors. In addition to the classification of injury mechanisms for each such class, suggestions for injury measures (IM) are given as well. They are specific injury measures which seem appropriate, useful and applicable to us for the classification and measurement of injury potentially occurring during physical human-robot interaction.

(1) e.g. represents blunt clamping in the near-singular configuration. As already shown, even for low-inertia robots this situation can become very dangerous and is therefore a possible serious threat with almost any robot on a fixed base within a (partially) confined workspace. Possible injuries are fractures and secondary injuries e.g. caused by penetrating bone structures or an injured neck if the trunk is clamped but the head is free. This would mean that the robot pushes the head further while the trunk remains in its position. Another possible threat is shearing off a locally clamped human along an edge. Appropriate indices are the contact force and CC. (2) is the clamped blunt impact in non-singular configuration. The injury potential is defined by the maximum joint torque $\boldsymbol{\tau}_{\max }$ and can range from no injury (as was shown for the LWRIII) to severe injury or even death for high-inertia (and joint torque)

\footnotetext{
$\overline{17}$ We consider only injuries for typical robot velocities and no hypothetic extreme cases. As shown in $[2,9]$ injury potential vastly increases with the impact velocity of the robot.
} 


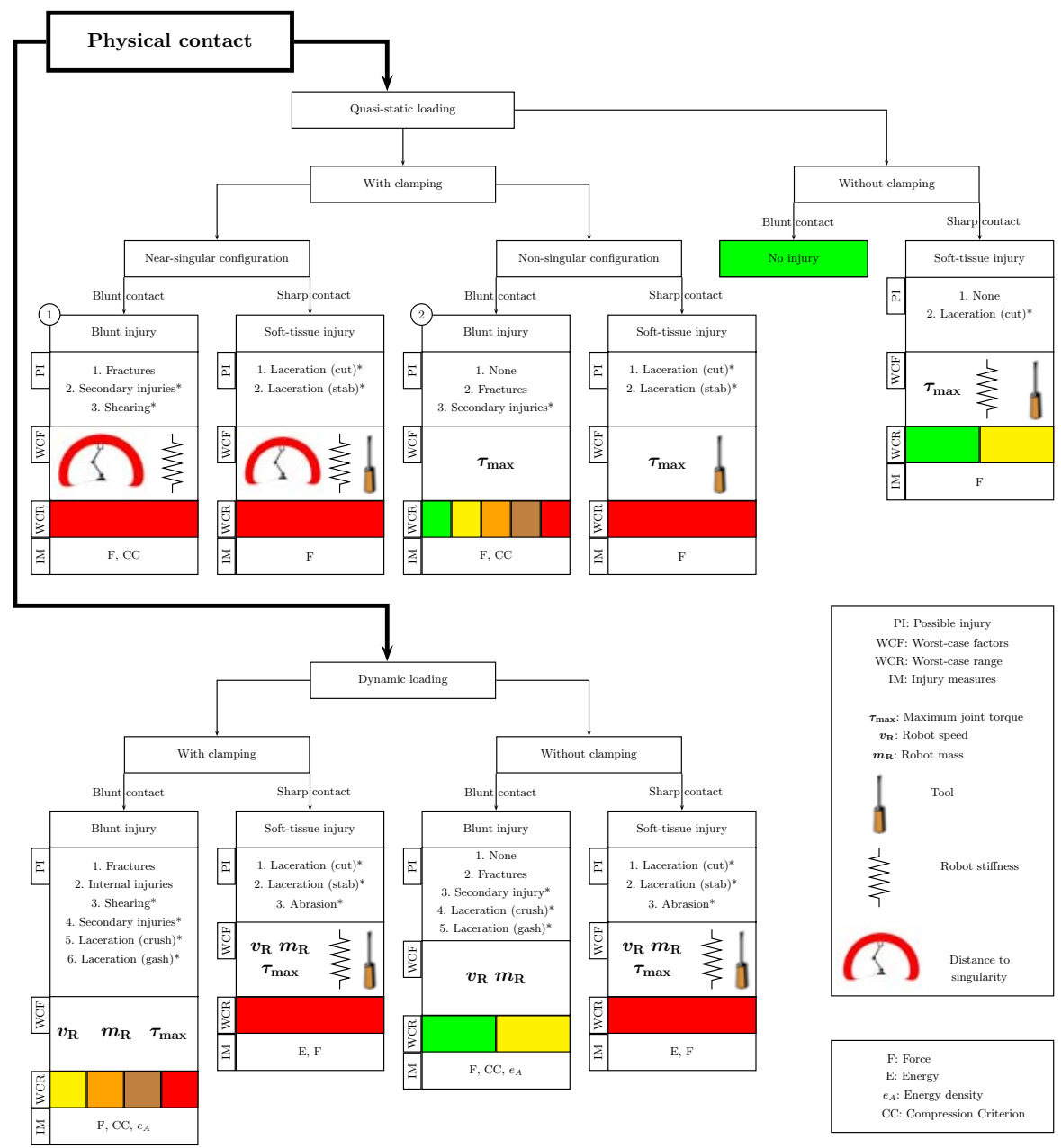

Fig. 5. Safety Tree showing possible injury (PI), major worst-case factors (WCF) and the possible worst-case range (WCR). * indicates still ongoing topics of research. Additionally, relevant injury criteria are given for the head, chest and soft-tissue injuries.

robots. The robot stiffness does not contribute to the worst-case since a robot without collision detection would simply increase the motor torque to follow the desired trajectory. Therefore, robot stiffness only contributes to the detection mechanism by enlarging the detection time. Also here, the contact force and the Compression Criterion are well suited to predict occurring injury.

\section{Conclusion}

In this paper we gave the first systematic evaluation and classification of possible injuries during physical human-robot interaction. We showed via experiment that potential injury of the head, occurring during a free impact, will saturate with increasing robot mass and from a certain mass on will only 
depend on the impact velocity. Generally blunt head or chest impacts without clamping at typical robot speed are, no matter how massive the robot is, definitely not life-threatening ${ }^{18}$. These are surprising and gratifying results and to our knowledge, they represent the first systematic experimental evaluation of possible injuries during robot-human impacts. Although e.g. HIC is not an appropriate measure of injury in robotics, other quite serious injuries of the head, as fractures of facial and cranial bones, are very likely to occur already at moderate velocities and seem to be a more relevant injury mechanism. The appropriate injury indicator for this class of injury is however not related to head acceleration but to impact forces.

Very different observations can be made in case of clamping which was evaluated with respect to robot mass and impact velocity. In case of clamping both the head and chest can be severely injured even leading to death, especially with increasing robot mass. Nevertheless, the low inertial properties of the LWRIII allow an impact velocity of up to $1 \mathrm{~m} / \mathrm{s}$ without leading to any of the investigated injuries.

Apart from the discussed dynamic impacts we showed that even low-inertia robots can become very dangerous in near-singular configurations in case of clamping.

Since soft-tissue injuries caused by sharp violence were to our knowledge not treated in the robotics literature up to now, an overview about relevant ones was outlined, showing which injury mechanisms still have to be investigated more in detail and especially what are possible tolerance values valuable for robotics.

Finally, we classified relevant injury mechanisms, important factors governing each injury process and the worst-case injury level emanating from it. This classification should be considered as a basis for further contributions, as well as a roadmap pointing out open issues and the variety of possible injury mechanisms in physical human-robot interaction.

Comparing the thresholds defined in ISO-10218 with our measurements it becomes clear that its definition is not based on biomechanical analysis. Such an evaluation leads to much higher tolerance values for blunt impacts. The intention of ISO-10218 is to keep the performance of the robot low in order to enable active avoidance of unintended contact by a human. If this is not possible very low exerted forces and power should avoid any kind of risk, i.e. ISO-10218 is a conservative safety requirement. However, this seems to be a strong restriction of robot performance while at the same time there is still lack of differentiated analysis. Especially tools and their corresponding injuries which would require even lower thresholds are not discussed. We suggest to define a more sophisticated and differentiated basis to achieve an optimal safety-performance tradeoff.

Videos illustrating and supporting key aspects proposed and explained in the paper are available for download at www.robotic.dlr.de/safe-robot.

\section{Acknowledgment}

This work has been partially funded by the European Commission's Sixth Framework Programme as part of the projects SMERobot ${ }^{\mathrm{TM}}$ under grant no. 011838 and PHRIENDS under grant no. 045359.

\footnotetext{
${ }_{18}$ Parts of the presented evaluation is carried out for average males.
} 


\section{References}

1. I. Asimov, The Caves Of Steel, A Robot Novel, 1954.

2. S. Haddadin, A. Albu-Schäffer, and G. Hirzinger, "Safety Evaluation of Physical Human-Robot Interaction via Crash-Testing," Robotics: Science and Systems Conference (RSS2007), 2007.

3. A. Bicchi and G. Tonietti, "Fast and Soft Arm Tactics: Dealing with the SafetyPerformance Trade-Off in Robot Arms Design and Control," IEEE Robotics \& Automation Mag., vol. 11, pp. 22-33, 2004.

4. M. Zinn, O. Khatib, and B. Roth, "A New Actuation Approach for Human Friendly Robot Design," Int. J. of Robotics Research, vol. 23, pp. 379-398, 2004.

5. ISO10218, "Robots for industrial environments - Safety requirements - Part 1: Robot," 2006.

6. K. Ikuta, H. Ishii, and M. Nokata, "Safety Evaluation Method of Design and Control for Human-Care Robots," Int. J. of Robotics Research, vol. 22, no. 5, pp. 281-298, 2003.

7. J. Heinzmann and A. Zelinsky, "Quantitative Safety Guarantees for Physical Human-Robot Interaction," Int. J. of Robotics Research, vol. 22, no. 7-8, pp. 479-504, 2003.

8. H.-O. Lim and K. Tanie, "Human Safety Mechanisms of Human-Friendly Robots: Passive Viscoelastic Trunk and Passively Movable Base," Int. J. of Robotics Research, vol. 19, no. 4, pp. 307-335, 2000.

9. S. Haddadin, A. Albu-Schäffer, and G. Hirzinger, "Multi-level Safety Analysis \& Design," Robotics: Science and Systems Workshop: Interfaces, devices and methods for robots adapted to Small and Medium Enterprises, 2008.

10. S. Haddadin, A. Albu-Schäffer, , M. Strohmayr, and G. Hirzinger, "Approaching Asimov's 1st Law II: The Impact of the Robot's Weight Class," VIDEO, submitted to: IEEE Int. Conf. on Robotics and Automation (ICRA2008), 2007.

11. S. Haddadin, A. Albu-Schäffer, and G. Hirzinger, "The Role of the Robot Mass and Velocity in Physical Human-Robot Interaction - Part I: Unconstrained Blunt Impacts," in submitted to: IEEE Int. Conf. on Robotics and Automation (ICRA2008), 2008.

12. - "The Role of the Robot Mass and Velocity in Physical Human-Robot Interaction - Part II: Constrained Blunt Impacts," in submitted to: IEEE Int. Conf. on Robotics and Automation 2008 (ICRA 2008), 2008.

13. A. De Luca, A. Albu-Schäffer, S. Haddadin, and G. Hirzinger, "Collision Detection and Safe Reaction with the DLR-III Lightweight Manipulator Arm," IEEE/RSJ Int. Conf. on Intelligent Robots and Systems (IROS2006), pp. 1623$1630,2006$.

14. S. Haddadin, A. Albu-Schäffer, A. De Luca, and G. Hirzinger, "Collision Detection \& Reaction tailored to safe physical Human-Robot Interaction," submitted to: IEEE Int. Conf. on Robotics and Automation (ICRA2008), 2008.

15. G. W. Nyquist, J. M. Cavanaugh, S. J. Goldberg, and A. I. King, "Facial Impact Tolerance and Response," SAE Paper No.861896, Proc. 30th Stapp Car Crash Conference, pp. 733-754, 1986.

16. D. L. Allsop, C. Y. Warner, M. G. Wille, D. C. Schneider, and A. M. Nahum, "Facial Impact Response-A Comparison of the Hybrid III Dummy and Human Cadaver," SAE Paper No.881719, Proc. 32th Stapp Car Crash Conference, pp. 781-797, 1988.

17. J. McElhaney, R. Stalnaker, and V. Roberts, "Biomechanical Aspects of Head Injury," Human Impact Response - Measurement and Simulation, 1972.

18. B. Brinkmann and B. Madea, Eds., Handbuch gerichtliche Medizin. Springer Verlag, 2004. 